\title{
High-speed Raman-encoded molecular imaging of freshly excised tissue surfaces with topically applied SERRS nanoparticles
}

\author{
Yu "Winston" Wang \\ Qian Yang \\ Soyoung Kang \\ Matthew A. Wall \\ Jonathan T. C. Liu
}




\title{
High-speed Raman-encoded molecular imaging of freshly excised tissue surfaces with topically applied SERRS nanoparticles
}

\author{
Yu "Winston" Wang, ${ }^{a, *, \dagger}$ Qian Yang, ${ }^{a, b, \dagger}$ Soyoung Kang, ${ }^{a}$ Matthew A. Wall, ${ }^{a, c}$ and Jonathan T. C. Liu"d, \\ aUniversity of Washington, Department of Mechanical Engineering, Seattle, Washington, United States \\ ${ }^{b}$ Chengdu Medical College, Collaborative Innovation Center of Sichuan for Elderly Care and Health, School of Pharmacy, Chengdu, China \\ 'Institute for Systems Biology, Seattle, Washington, United States \\ dUniversity of Washington, School of Medicine, Department of Pathology, Seattle, Washington, United States
}

\begin{abstract}
Surface-enhanced Raman scattering (SERS) nanoparticles (NPs) are increasingly being engineered for a variety of disease-detection and treatment applications. For example, we have previously developed a fiber-optic Raman-encoded molecular imaging (REMI) system for spectral imaging of biomarker-targeted SERS NPs topically applied on tissue surfaces to identify residual tumors at surgical margins. Although accurate tumor detection was achieved, the commercial SERS NPs used in our previous studies lacked the signal strength to enable high-speed imaging with high pixel counts (large fields of view and/or high spatial resolution), which limits their use for certain time-constrained clinical applications. As a solution, we explored the use of surface-enhanced resonant Raman scattering (SERRS) NPs to enhance imaging speeds. The SERRS NPs were synthesized de novo, and then conjugated to HER2 antibodies to achieve high binding affinity, as validated by flow cytometry. Under identical tissue-staining and imaging conditions, the targeted SERRS NPs enabled reliable identification of HER2-overexpressed tumor xenografts with 50-fold-enhanced imaging speed compared with our standard targeted SERS NPs. This enables our REMI system to image tissue surfaces at a rate of $150 \mathrm{~cm}^{2}$ per minute at a spatial resolution of $0.5 \mathrm{~mm}$. ๑ 2018 Society of Photo-Optical Instrumentation Engineers (SPIE) [DOI: 10.1117/1.JBO.23.4.046005]
\end{abstract}

Keywords: biomedical optics; molecular imaging; surgical guidance; Raman spectroscopy; nanomedicine.

Paper 180024R received Jan. 13, 2018; accepted for publication Mar. 26, 2018; published online Apr. 16, 2018.

\section{Introduction}

Surgery remains the most popular first-line treatment strategy for the majority of solid tumors. However, in many cases, local recurrence occurs due to incomplete tumor resection (i.e., positive margins). Examples include breast conserving surgery (a.k.a. lumpectomy, $20 \%$ to $50 \%$ of positive margin rates) ${ }^{1}$ radical prostatectomy ( $11 \%$ to $38 \%$ of positive margin rates), ${ }^{2}$ and head and neck tumor resections (11\% to $16 \%$ of positive margin rates). ${ }^{3-5}$ Complete resection of tumors in a single operation is important for minimizing patient trauma (physical and emotional) and reducing health care costs. Molecular imaging techniques have been investigated as a highly specific means to visualize tumors during surgery for the purposes of maximizing tumor resection while preserving important benign tissues. ${ }^{6}$ However, a major hurdle for molecular-image-guided surgery is the molecular heterogeneity of tumors amongst various patients as well as within individuals. ${ }^{7}$ One strategy that has been explored to mitigate the effects of this molecular heterogeneity has been to evaluate a diverse panel of biomarkers through multiplexed molecular imaging.

Surface-enhanced Raman-scattering (SERS) nanoparticles (NPs) have attracted interest for molecular imaging applications due to their excellent multiplexing capabilities. ${ }^{8,9}$ These SERS

*Address all correspondence to: Yu "Winston" Wang, yuwang2@uw.edu; Jonathan T. C. Liu, jonliu@uw.edu

TThese authors contributed equally to this work.
NPs are available in many "flavors," each of which emits a characteristic Raman "fingerprint" spectrum when illuminated at a common wavelength. ${ }^{10}$ By functionalizing various flavors of SERS NPs with different targeting molecules (e.g., antibodies, ${ }^{11}$ affibodies, ${ }^{12}$ and aptamers ${ }^{13}$ ), the NPs can be multiplexed to simultaneously target and image a large panel of cancer biomarkers. Utilizing commercial SERS NPs, we have recently developed an intraoperative imaging technique to rapidly image multiple biomarkers at the margins of freshly resected breast tissues. ${ }^{11,14-16}$ This intraoperative technique circumvents toxicity and sterility concerns by staining and imaging fresh surgical specimens ex vivo, and utilizes ratiometric imaging of targeted versus untargeted NPs to mitigate the misleading effects of nonspecific NP accumulation and to achieve unambiguous biomarker quantification. ${ }^{14,17,18}$ We have shown that after 5 min of staining, and $10 \mathrm{~s}$ of rinsing, followed by raster-scanned spectral imaging of tissue surfaces ( $<15 \mathrm{~min}$ in total), four biomarkers can be simultaneously quantified across a surgical margin surface, which has enabled this technique to achieve $89.3 \%$ sensitivity and $92.1 \%$ specificity for the detection of breast tumors. ${ }^{16}$ This intraoperative technique possesses several advantages over conventional frozen-section and formalin-fixed paraffinembedded (FFPE) histopathology but can also complement these methods, because it is nondestructive (no physical sectioning is needed) and does not interfere with downstream pathology. ${ }^{14,16}$ For example, by comprehensively imaging an

1083-3668/2018/\$25.00 @ 2018 SPIE 
(a) Synthesis

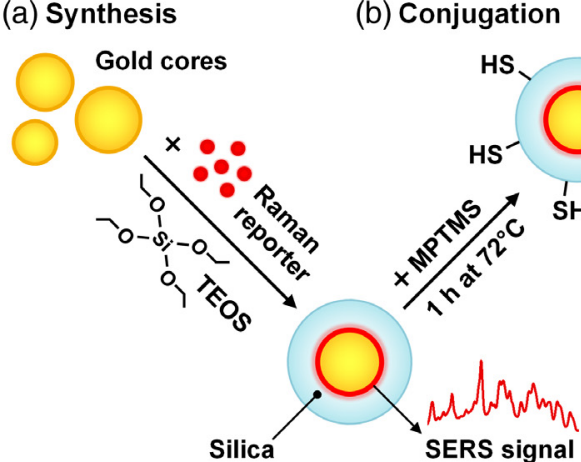

\section{(b) Conjugation}<smiles>CCS</smiles><smiles>C1C[AsH]C[SiH2]1</smiles>
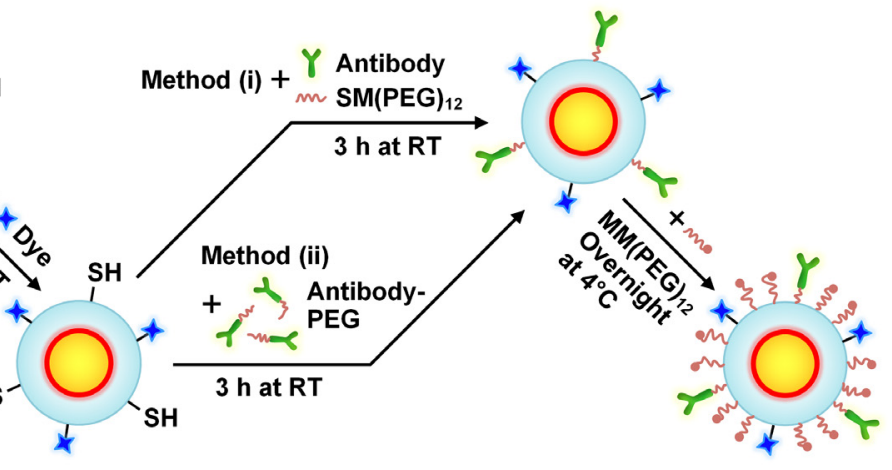

Fig. 1 (a) Schematic illustration of the synthesis of SERRS NPs and (b) conjugation of the NPs to monoclonal antibodies. Two conjugation methods were tested in this study such as (i) antibodies, bifunctional PEG crosslinkers $\left[\mathrm{SM}(\mathrm{PEG})_{12}\right]$, and thiolated NPs were reacted altogether (referred to as the one-step method); (ii) antibodies were first reacted with the bifunctional PEG crosslinkers to form antibody-PEG conjugates, and then reacted with the thiolated NPs to produce antibody-NP conjugates (referred to as the two-step method).

entire surgical margin surface, this technique can overcome the sampling limitations associated with slide-based histology, in which tissues are sectioned in the vertical direction at periodic intervals with minimal sampling of the tissue surfaces (i.e., the inked margin). Although the translational potential of this technique to guide tumor-resection procedures has been investigated, the commercial SERS NPs used in our previous studies lacked the signal strength to enable high-speed imaging with high-pixel-counts (large fields of view and/or high spatial resolution), which limits their use for imaging large tissue specimens intraoperatively.

Surface-enhanced resonant Raman scattering (SERRS) NPs have been developed in recent years, featuring high brightness. ${ }^{19}$ Similar to SERS NPs, SERRS NPs consist of a gold core that provides plasmonic enhancement of the RS generated by a layer of Raman reporters adsorbed onto the surface of the gold cores. A silica coating is used to isolate the Raman-scattering chemicals from the environment such that the NPs emit a stable fingerprint spectrum that is not sensitive to their surrounding environment. The silica shell also prevents the NPs from aggregating. Unlike SERS NPs, SERRS NPs utilize Raman reporters with absorption peaks that are matched with the NIR light sources that are typically used for excitation (e.g., 785-nm diode lasers), which significantly enhances the SERS signals and allows the NPs to be detected at fM concentrations. ${ }^{19-21}$ Molecular imaging of tissues using biomarker-targeted SERRS NPs has been reported by others. ${ }^{13,20}$ For example, studies using commercial Raman microscopes have shown that a $1-\mathrm{cm}^{2}$ tissue area can be imaged with microscopic resolution $(14 / 200 \mu \mathrm{m}$ in the $x / y$ directions) in $<1 \mathrm{~h}^{13,20}$ However, rapid molecular imaging of large tissues ( $>5 \mathrm{~cm}^{2} / \mathrm{min}$ ) using SERRS NPs has not been demonstrated, which is key for intraoperative use. Here, we explored the use of SERRS NPs to enhance the imaging speed of a fiber-optic spectral imaging system we previously developed for rapid intraoperative molecular imaging of large tissue areas $\left(>5 \mathrm{~cm}^{2} / \mathrm{min}\right)$. To facilitate the evaluation of the SERRS NPs, the commercial SERS NPs (purchased from BD) used in our previous studies were included in this study for a side-by-side comparison of their spectral intensities/ shape, detection limits, biomarker-targeting abilities, and imaging speeds.

\section{Materials and Methods}

\subsection{Synthesis of Surface-Enhanced Resonant Raman Scattering Nanoparticles}

The SERRS NPs were synthesized via a seed-mediated approach, based on a published protocol (with a few modifications). ${ }^{21,22}$ In brief, gold nanospheres were synthesized by adding $7.5-\mathrm{mL}(1 \% \mathrm{w} / \mathrm{v})$ sodium citrate to $1-\mathrm{L}(0.25 \mathrm{mM})$ boiling $\mathrm{HAuCl}_{4}$, and allowed to react for $1 \mathrm{~h}$ with 300 -rpm stirring. The as-synthesized gold nanospheres were concentrated by centrifugation $\left(7500 \mathrm{~g} \times 10 \mathrm{~min}\right.$ at $4^{\circ} \mathrm{C}$ with $50-\mathrm{mL}$ centrifuge tubes) and dialyzed for 5 days $(3.5 \mathrm{kDa}$ molecular weight cutoff, Thermo Fisher Scientific). For the addition of the Raman reporter and silica coating [Fig. 1(a)], the obtained gold nanospheres $(70 \mu \mathrm{L}$ at $2 \mathrm{nM})$ were added to $1-\mathrm{mL}$ absolute ethanol in the presence of $50-\mu \mathrm{L}$ tetraethyl orthosilicate (333859, Sigma Aldrich), 20- $\mu \mathrm{L} \mathrm{28 \%} \mathrm{ammonium} \mathrm{hydroxide} \mathrm{(338818,} \mathrm{Sigma}$ Aldrich), $2-\mu \mathrm{L}$ Raman reporter (25 mM, IR792 or IR780) in DMF, and allowed to react for 25 min with stirring. The absorption peaks of the Raman reporter dyes, IR780 and IR792, are at $781 \mathrm{~nm}$ (576409, Sigma Aldrich) and $792 \mathrm{~nm}$ (425982, Sigma Aldrich), respectively. The SERRS NPs were isolated by centrifugation $(5000 \mathrm{rpm} \times 20 \mathrm{~min})$ and washing them $3 \times$ with ethanol, and then stored in ethanol until thiolation.

\subsection{Conjugation of Nanoparticles to Monoclonal Antibodies}

The SERRS NPs were functionalized with monoclonal antibodies [Fig. 1(b)] for molecular imaging experiments. Prior to conjugation, the SERRS NPs were modified with $1 \%$ (3-mercaptopropyl) trimethoxysilane (Sigma-Aldrich) to introduce free thiols $(-\mathrm{SH})$ on the NP surface (details can be found in Ref. 19). The SERS NPs, which were already functionalized with active thiols at the time of purchase, were directly used for conjugation. Based on our previously developed protocol, ${ }^{17}$ the thiolated NPs were first reacted with a fluorescent dye, DyLight 650 Maleimide (Thermo Scientific, 62295), for the purposes of flow-cytometry characterization. The NPs were then conjugated with either an anti-HER2 (Thermo Scientific, MS229PABX) or an isotype-control antibody (Thermo 
Scientific, 110407) at 500 molar equivalents per NP, in the presence of a heterobifunctional PEG crosslinker SM(PEG) 12 (Thermo Scientific, 22112) at $1.5 \times 10^{4}$ molar equivalents per NP [Fig. 1(b), method i]. Finally, excess $\mathrm{MM}(\mathrm{PEG})_{12}$ at $6 \times 10^{5}$ molar equivalents per NP (Thermo Scientific, 22711) was added to the NPs and reacted overnight at $4^{\circ} \mathrm{C}$, to block residual thiols on the NPs and reduce nonspecific accumulation of the NPs. ${ }^{17,23}$

\subsection{Spectral Imaging System}

A customized spectral-imaging system has been developed to image SERS/SERRS NPs that are topically applied on tissue specimens. ${ }^{14}$ In brief, a $785-\mathrm{nm}$ laser (maximum $50 \mathrm{~mW}$ ) is used to illuminate the samples via a multimode fiber $(100-\mu \mathrm{m}$ core, $0.10 \mathrm{NA}$ ), creating a submillimeter-diameter laser spot (imaging resolution). Raman-scattered photons from illuminated NPs are collected by 27 multimode fibers $(200-\mu \mathrm{m}$ core, $0.22 \mathrm{NA}$ ) and transmitted to a customized spectrometer (Andor Holospec), where they are dispersed onto a cooled deep-depletion spectroscopic CCD (Andor Newton DU920PBR-DD). For raster-scanned tissue imaging, a two-axis translation stage was constructed and controlled through a custom LabVIEW program. ${ }^{14}$ Raster-scanned imaging of entire tissue specimens was performed by holding the position of the imaging probe constant and scanning the tissue with respect to the probe.

A direct classical least squares (DCLS) algorithm is employed to demultiplex the collected spectra and to calculate the concentrations of the NPs as described previously. ${ }^{14,15}$ In brief, other than sources of random noise, it is assumed that each measured spectrum consists of a linear combination of individual NP spectra and broadband background signals from tissues, buffers, and substrate materials. As the reference spectra of tissues can vary between sites due to differences in optical and chemical properties, background tissue spectra are first acquired, from which a principal components analysis is performed. This has been shown to be a robust method to accommodate for variations in the background spectra. ${ }^{14,24-26}$ In other words, before staining the human breast sample, the entire tissue is raster-scanned to obtain a complete set of background spectra (100 to 400 spectra depending on the tissue size). The acquired background spectra are then analyzed to calculate the first three principal components and an average background spectrum, which are all used as background references with the ability to cumulatively account for almost all of the observed background variations. After NP staining and imaging, the spectra acquired from stained tissues are demultiplexed using the DCLS algorithm to calculate the weight of each spectral component (e.g., the different flavors of NPs, the average tissue background, and principal components of the tissue background). The NP concentrations and concentration ratios are calculated based on calibration measurements with stock NPs of known concentrations.

\subsection{Imaging Procedures}

For linearity measurements, and for determining the limit of detection of spectral measurements [Figs. 2(e)-2(h)], the NP samples were prepared by mixing two NP flavors in a $1: 1$ ratio and diluting the mixture to different concentrations. About $20 \mu \mathrm{L}$ of each sample were placed in a customized well plate (refer to Fig. S1 in Ref. 11) for spectral measurements, where three spectral measurements of each sample were acquired with the fiber-optic imaging system. The acquired spectra were then demultiplexed to determine the individual (a)

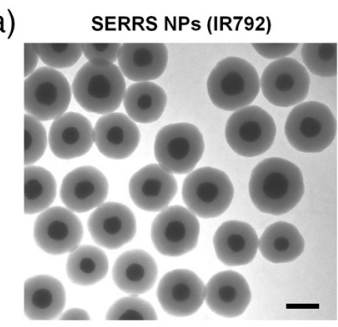

(b)

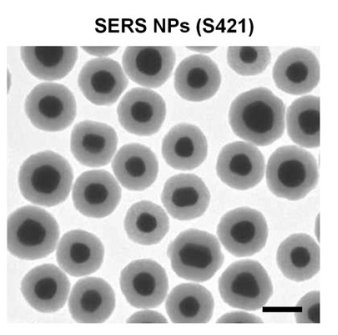

(c)
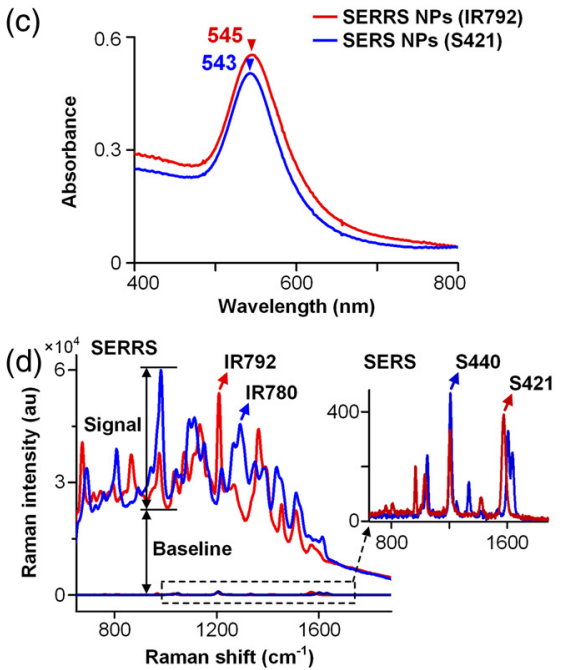
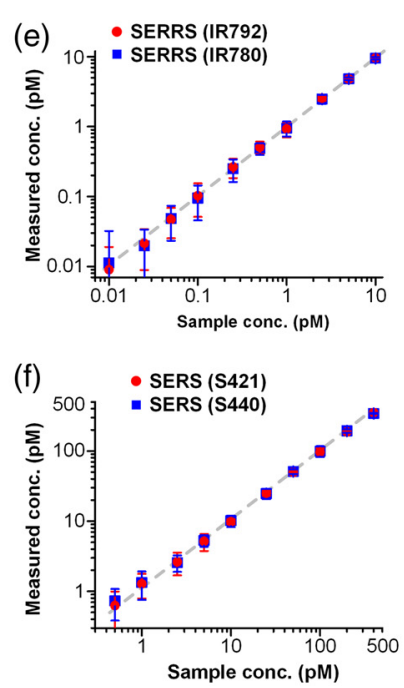

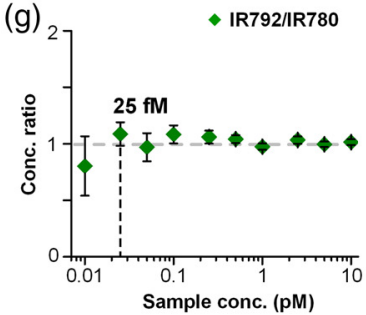

(h)

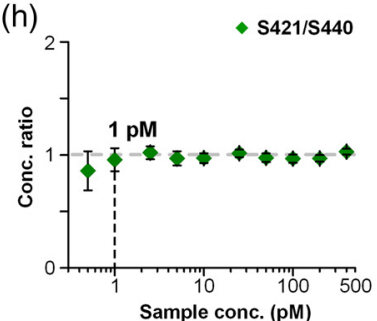

Fig. 2 Comparison of SERRS NPs and commercial SERS NPs based on their structure, spectrum, and detection limits. (a, b) TEM images (acquired using Philips CM100 TEM). The gold cores of the NPs appear black, and are encapsulated by a silica shell. Scale bars $=100 \mathrm{~nm}$. (c) Absorption spectra of SERRS NPs and SERS NPs. Note that the absorption cross section of the Raman reporters is negligible compared with that of the gold cores (which dominate the light absorption). ${ }^{21}$ (d) Spectra of the NPS (SERRS and SERS) used in this study. All NP samples were measured under the same conditions (20-pM NPs, 18-mW laser power, 0.5-s integration time). (e-h) Linearity measurements that are used to determine the limit of detection. For each NP sample, three spectral measurements were acquired with the fiber-optic imaging system. The acquired spectra were then demultiplexed to determine the individual NP concentrations (e, f) and the concentration ratios $(g, h)$. For this study, the limit of detection [marked with the dashed lines in plots $(\mathrm{g})$ and $(\mathrm{h})$ ] was defined as the NP concentration at which the error in the measured NP concentration ratios surpassed $15 \%$. 
NP concentrations [Figs. 2(e) and 2(f)] and the concentration ratios [Figs. 2(g) and 2(h)]. Refer to Sec. 2.3 for spectral demultiplexing and concentration measurement methods.

ZR-75-1 tumor xenografts and normal tissue (muscle) specimens were used for the tissue-imaging experiments (Fig. 4). Unstained specimens were first imaged to acquire a set of background spectra for the calculation of their principal components (to account for the tissue background during least squares demultiplexing). The specimen surfaces were then topically stained with the NP staining solution (SERS or SERRS NPs) using a convection-enhanced staining method. ${ }^{11}$ Based on a previously optimized staining protocol, ${ }^{14}$ the staining solution consisted of a mixture of HER2-NPs and isotype-NPs (150 pM per flavor) supplemented with $1 \%$ BSA to minimize nonspecific binding. After $5 \mathrm{~min}$ of staining, the tissue specimens were rinsed in 50-mL PBS with gentle agitation for $10 \mathrm{~s}$. Four stained specimens, including two specimens stained with the SERRS NPs and another two specimens stained with the SERS NPs, were then placed on a glass slide for raster-scanned imaging of the stained tissue surfaces. The spectral measurement conditions (e.g., working distance, laser power) were the same for all NP measurements. Three scans were performed continuously using 100-, 10-, and 2-ms integration times, followed by spectral demultiplexing and ratiometric mapping (Fig. 4). To avoid detector saturation from the SERRS NP signals at an integration time of $100 \mathrm{~ms}$, the laser power was reduced to $2 \mathrm{~mW}$. To improve pixel coregistration between images, a small scanning area $\left(26 \times 22 \mathrm{~mm}^{2}\right)$, short integration times, and a large laser spot $(500 \mu \mathrm{m}$, i.e., the spatial resolution) were used to reduce the total imaging time and to minimize the effects of tissue deformation (e.g., due to dehydration) during the scans.

\subsection{Cell Culture and Flow Cytometry}

The two cell lines employed in this study were $\mathrm{SkBr} 3$ (ATCC, HTB-30D) and ZR-75-1 (ATCC, CRL-1500), both of which overexpress HER2 $2{ }^{27} \mathrm{SkBr} 3$ cells were cultured in McCoy's 5A medium (Lonza, 12-688F), and ZR-75-1 cells were cultured in RPMI-1640 medium (ATCC, 30-2001), both of which were supplemented with $10 \%$ fetal bovine serum (FBS, Thermo Scientific, SH3008803) and 1\% penicillin-streptomycin (Lonza, 17-602E). All cells were cultured at $37^{\circ} \mathrm{C}$ with $5 \% \mathrm{CO}_{2}$. Trypsin EDTA 1X (Mediatech, MT25051CI) was used to detach cells.

Flow cytometry: for the SERRS NPs and SERS NPs, HER2NPs and isotype-NPs (150 pM) were individually used to stain $\mathrm{SkBr} 3$ cells ( 0.25 million) in $50-\mu \mathrm{L}$ FACS buffer (20\% FBS in PBS) for 15 min (at RT, with 300-rpm agitation), followed by three rounds of purification via centrifugation ( $400 \mathrm{~g}$ for $5 \mathrm{~min}$ ) and supernatant-replacement (500- $\mu$ L FACS buffer per rinse). The stained cells were then analyzed via flow cytometry using BD LSR II flow cytometer. Figures 3(a) and 3(b) show the fluorescence histograms from cells stained with each NP flavor.

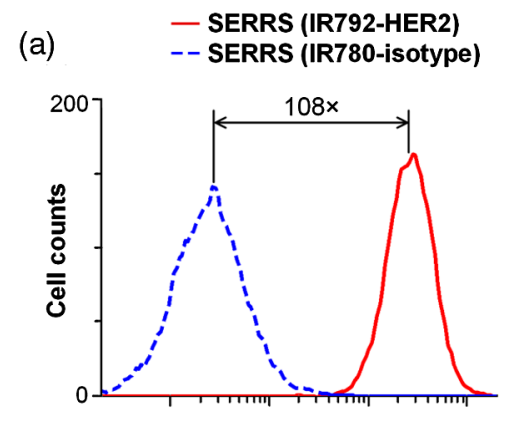

Fluorescence intensity

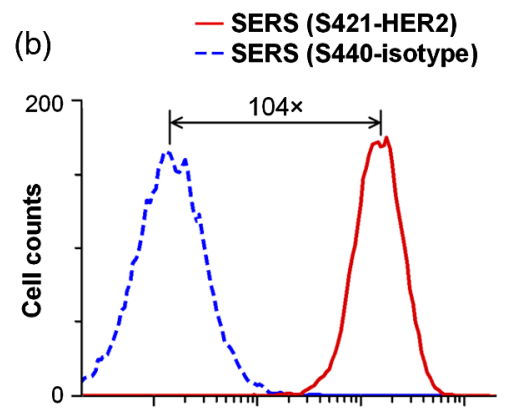

Fluorescence intensity

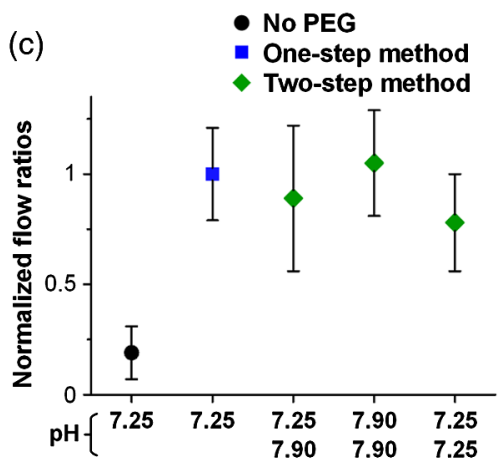

(d) SERRS (792-HER2)

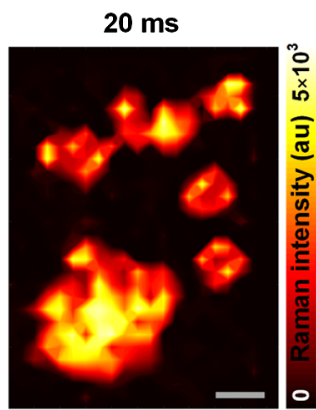

(e) SERS (421-HER2)
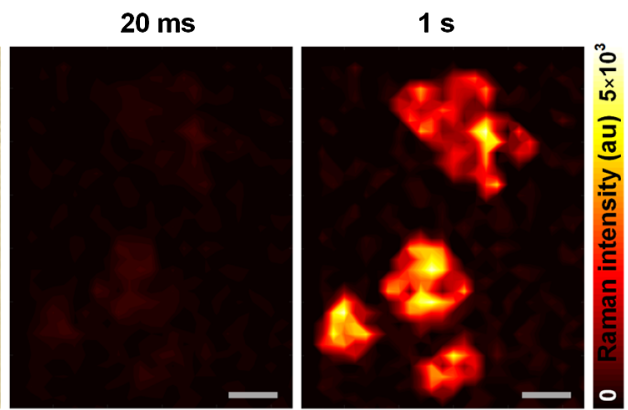

Fig. 3 Validation of the binding affinity of targeted NPs to membrane receptors on cultured cells. (a, b) Flow cytometry validation. Fluorescence histograms are shown from cells stained with each NP flavor. (c) Comparison of the ratio of targeted versus untargeted NPs (based on flow cytometry) for NPs that were conjugated using one-step or two-step methods. For each condition, three batches of conjugated NPs were prepared to quantify the results. (d, e) Microscopic imaging (Renishaw InVia Raman microscope) of SkBr3 cells stained with HER2-NPs (flow cytometry samples). The NP-stained cells were fixed in formalin and embedded in $1 \%$ agarose to immobilize them for imaging. The samples were imaged with a raster-scanned laser spot that measured 5 microns in diameter, with Raman signals collected through a high-NA (0.8) objective with a confocal pinhole applied, yielding an axial sectioning thickness of $\sim 5 \mu \mathrm{m}$. The scale bars represent $20 \mu \mathrm{m}$. 


\subsection{Mouse Xenograft Model}

Nude mice (Taconic Farms Inc., model NCRNU-F) were used to develop ZR-75-1 tumor xenografts. All animal procedures were approved by the Institutional Animal Care and Use Committee at the University of Washington (Protocol 4345-01). ZR-75-1 cancer cells $\left(3 \times 10^{6}\right)$ were suspended in Matrigel (BD biosciences, 354234) at a $1: 1$ volume ratio at $4^{\circ} \mathrm{C}$ to form a $100-\mu \mathrm{L}$ mixture. Nude mice (5 to 8 weeks) were subcutaneously implanted in their flanks with the cell-Matrigel mixture. The mice were euthanized by $\mathrm{CO}_{2}$ inhalation when the tumors reached a size of 8 to $10 \mathrm{~mm}$, followed by the surgical removal of the implanted tumors.

\subsection{Statistical Analysis}

Statistical analyses were performed in MATLAB or Origin. For the plots in Figs. 2(e)-2(h) and 3(c), the error bars represent the standard deviation of the results. For the box plots in Fig. 4(o), the bottom and top bars represent the 5th and 95th percentiles of the data, respectively, the bottom and top of the box represent the first and third quartiles of the data respectively, and the band inside the box represents the median (second quartile) of the data.

\section{Results and Discussion}

\subsection{Structure, Spectrum, and Detection Limits of the SERRS/SERS Nanoparticles}

The synthesized SERRS NPs and the commercial SERS NPs have the same sandwich structure and similar sizes [Figs. 2(a) and 2(b)]. By synthesizing SERRS NPs with silica coatings of different thicknesses (15 to $45 \mathrm{~nm}$ ), we found that NPs with a 45-nm coating thickness produced the most stable signals. Therefore, SERRS NPs with an overall size of $150 \mathrm{~nm}$ were used in this study.
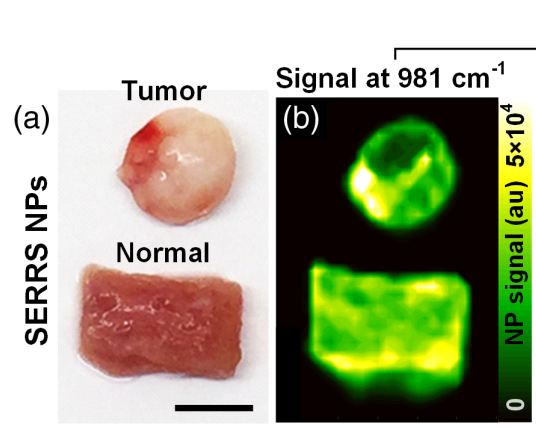

$100 \mathrm{~ms}$

Ratio (792/780)
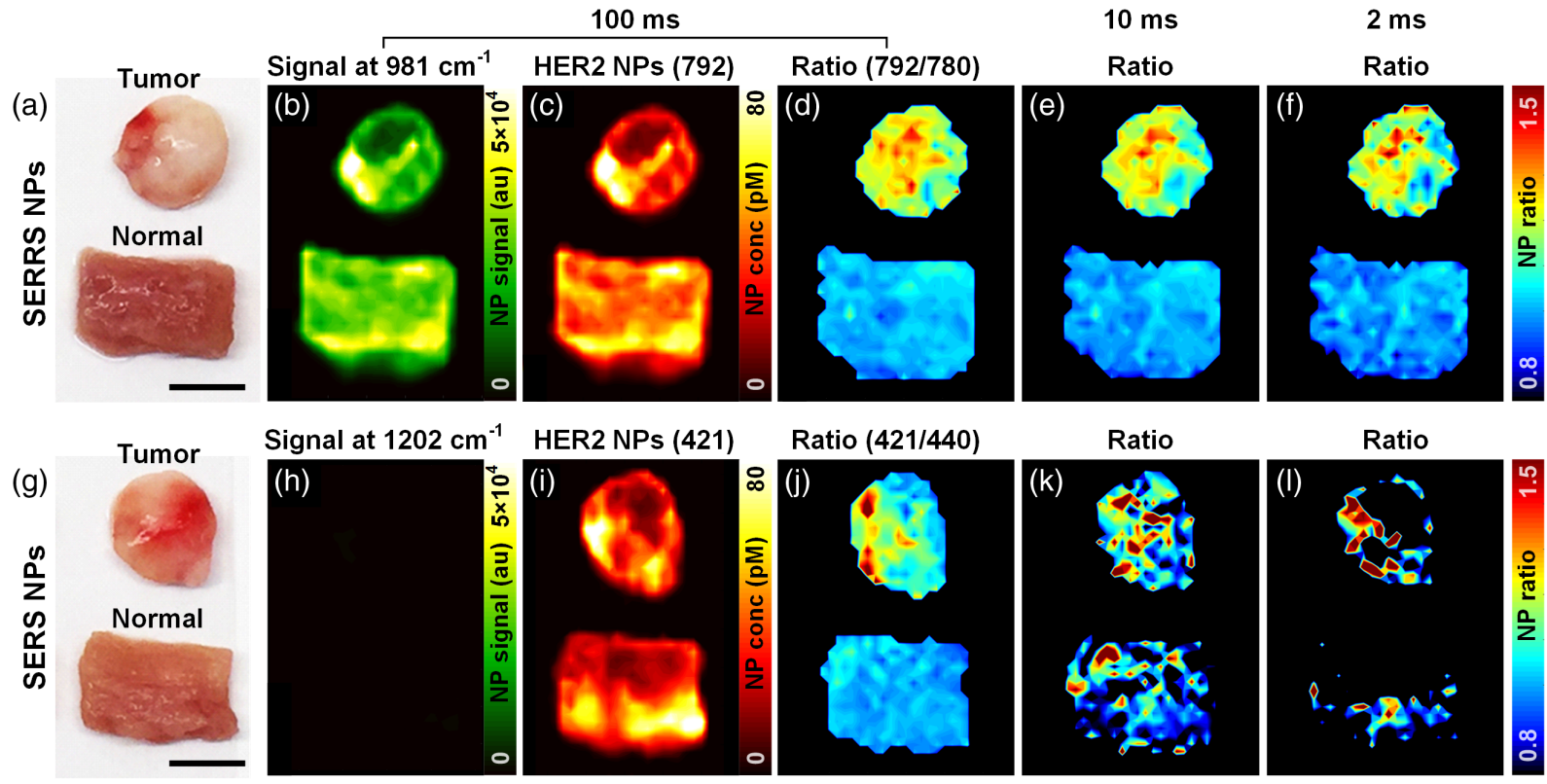

Signal at $1202 \mathrm{~cm}^{-1}$

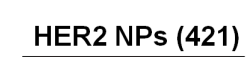

Ratio (421/440)
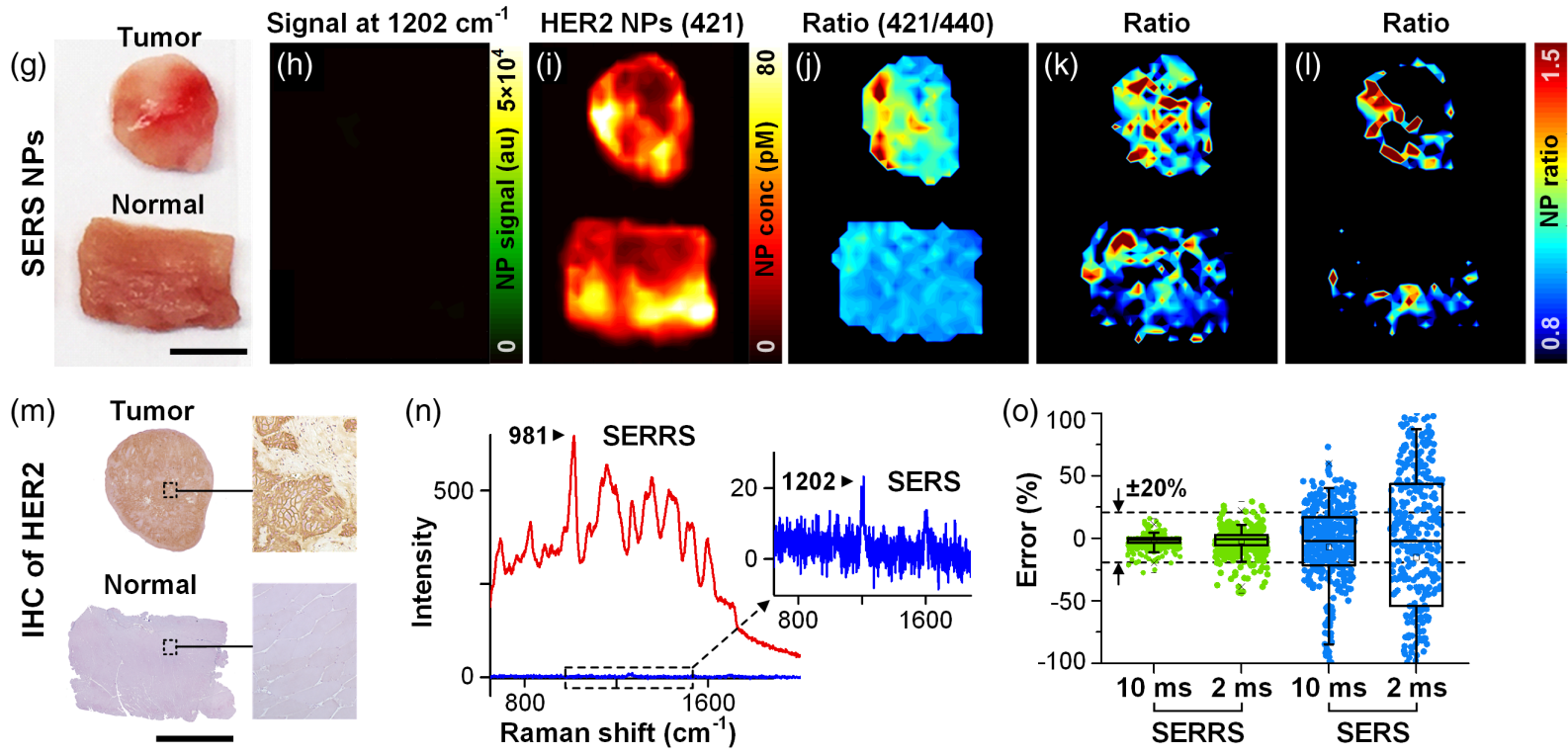

Fig. 4 SERRS NPs enable high-speed ratiometric imaging of HER2 in tissues. Three scans were performed at different integration times: (b-d, h-j) $100 \mathrm{~ms},(\mathrm{e}, \mathrm{k}) 10 \mathrm{~ms}$, and (f, I) $2 \mathrm{~ms}$. The SERRS NPs were used for (a-f), and the SERS NPs were used for $(g-l)$. $(a, g)$ Photographs of resected ZR-75-1 tumor xenografts (HER2-positive) and normal tissues (muscle). (b, h) Images showing the signal intensity at $981 \mathrm{~cm}^{-1}$ (major SERRS peak) and $1202 \mathrm{~cm}^{-1}$ (major SERS peak), as indicated in panel $n$. (c, i) The absolute concentration of the targeted NPs is shown, in which there is ambiguity between chemically specific NP accumulation and nonspecific sources of NP accumulation. (d, j) The concentration ratio of the HER2-NPs versus isotype-NPs (specific veresus nonspecific NP retention), a measure that correlates with biomarker expression levels, as validated by $(\mathrm{m})$ immunohistochemistry (IHC for HER2). (n) Raw spectra acquired at a 2-ms integration time (SNR $\sim 40$ for SERRS NPs and SNR $\sim 1.5$ for SERS NPs). Representative spectra were acquired from bright tissue regions stained with SERRS and SERS NPs. (o) With images (d) and (j) serving as gold standards, the measurement errors for all of the pixels in images $(\mathrm{e}),(\mathrm{f}),(\mathrm{k})$, and (l) are plotted. The scale bars represent $5 \mathrm{~mm}$. 
The spectra generated by the SERS NPs and SERRS NPs were measured with our previously developed fiber-optic Raman-encoded molecular imaging (REMI) system. ${ }^{14}$ Compared with the SERS NPs, the SERRS NPs showed $\sim 100 \times$ enhanced signal strength [Fig. 2(d)], which allowed them to achieve good detection linearity $(<15 \%$ error) down to the $\mathrm{fM}$ range and a two-flavor limit of detection (25 fM per NP flavor) that was $40 \times$ lower [Figs. 2(e)-2(h)]. One advantage of the SERS NPs is that their spectra are cleaner, with well-separated Raman peaks and a negligible baseline [Fig. 2(d)], which may facilitate their use for highly multiplexed molecular imaging (e.g., demultiplexing of 5 to $10 \mathrm{NP}$ flavors has been reported. ${ }^{10,16}$ ). Dense multiplexing was not explored in this initial study, in which a maximum of two NP flavors were multiplexed (sufficient for accurate ratiometric imaging of a single biomarker target). However, future studies should be performed to investigate the ability to perform heavily multiplexed molecular imaging with SERRS NPs that produce spectra that are more congested in comparison with the SERS NPs used in our previous studies.

To ensure reproducible imaging, TEM images and Raman spectra were acquired after each batch of SERRS NPs was synthesized. The NPs were used for imaging experiments only when their size, purity, and structural uniformity were similar to what is shown in Fig. 2(b), and their Raman signal intensities were similar to what is shown in Fig. 2(d) ( $\pm 20 \%$ error).

\subsection{Rapid Molecular Imaging of Cultured Cells Using Targeted SERRS/SERS Nanoparticles}

A HER2-positive cell line, SkBr3, was used to validate the specific binding ability of conjugated NPs via flow cytometry. As shown in Fig. 3(a), the cells stained with HER2-targeted SERRS NPs showed $100 \times$ enhanced fluorescence signal than the cells stained with the isotype-control SERRS NPs, suggesting a high binding affinity that is comparable with the HER2-targeted SERS NPs used in our previous studies [Fig. 3(b)].

Other than the aforementioned method of conjugating SERS NPs to antibodies (method i in Sec. 2.2), another conjugation method was reported by others (method ii) for conjugating SERRS NPs to antibodies, which enabled specific binding of targeted SERRS NPs to folate receptors in tissues. ${ }^{20}$ In this study, the effectiveness of these two methods were compared. For method ii, instead of mixing the antibody, $\mathrm{SM}(\mathrm{PEG})_{12}$, and NPs all together [method i in Fig. 1(b)], the antibodies were first incubated with a 10 -fold molar excess of SM(PEG) ${ }_{12}$ in 10-mM MOPS buffer ( $\mathrm{pH} 7.25$ or 7.90 ) for 30 min, purified with a centrifugal filter (100 kDa molecular weight cutoff, Sigma-Aldrich, Z677906) to remove unreacted SM(PEG) ${ }_{12}$, and then the antibody-PEG conjugates were added to the NPs in 10-mM MOPS buffer ( $\mathrm{pH} 7.25$ or 7.90 ) at 500 molar equivalents per NP [method ii in Fig. 1(b)]. This two-step method ensures that the $\mathrm{SM}(\mathrm{PEG})_{12}$ linkers are first bound to an antibody prior to being bound to the NPs, and provides the flexibility to individually optimize the conditions (e.g., $\mathrm{pH}$ ) for each individual reaction (PEG with antibody and PEG with thiolated NPs).

The two conjugation methods were compared based on the flow cytometry results [Fig. 3(c)]. Here, the flow cytometry ratio [normalized based on the ratio in Fig. 3(a)] was used as a surrogate measure related to the binding potential of the SERRS NPs on tissues. ${ }^{18}$ For the two-step method, the two $\mathrm{pH}$ values listed in Fig. 3(c) were used for the PEG-antibody reaction (top $\mathrm{pH}$ ) and PEG-NP reaction (bottom pH). The "no PEG" result reveals the low binding affinity of the NPs when the $\mathrm{SM}(\mathrm{PEG})_{12}$ linker is omitted from the conjugation (the antibodies are bound nonspecifically to the NP surface), which serves as a control for the other conditions. The results in Fig. 3(c) show that the one-step method [method i in Fig. 1(b)] and two-step method [pH 7.90/7.90, method ii in Fig. 1(b)] yielded similar results. Therefore, the simpler one-step method was used exclusively in subsequent parts of this study.

After flow cytometry, the cell samples were imaged with a commercial Raman microscope (Renishaw InVia). As shown in Figs. 3(d) and 3(e), the HER2-targeted SERRS NPs bound on $\mathrm{SkBr} 3$ cells exhibited much brighter Raman signals than the HER2-targeted SERS NPs bound on cells, enabling the system to image 3000 pixels/min (compared with 60 pixels / min for the SERS NPs). Such improvement in imaging speed could potentially enable real-time imaging of cells with subcellular resolution, for example, for monitoring physiological processes, studying NP binding and internalization, and other applications.

\subsection{Rapid Molecular Imaging of Tissues Using Multiplexed SERRS/SERS Nanoparticles}

Finally, the conjugated SERRS NPs and SERS NPs were used for molecular imaging of tissues, to compare their imaging speeds using our fiber-optic REMI system. ${ }^{14,16}$ For each type of NPs, a mixture of HER2-NPs and isotype-NPs (150 pM/flavor) was used to stain ZR-75-1 tumor xenografts and normal tissues (muscle) for $5 \mathrm{~min}$, using a convection-enhanced staining method, ${ }^{11}$ followed by a $10-\mathrm{s}$ rinse in PBS. The specimens stained with SERRS NPs exhibited much stronger signals than the specimens stained with SERS NPs [Figs. 4(b) and 4(h)]. However, calibrated measurements revealed that the absolute concentrations of the SERRS and SERS NPs in the tissues were similar [Figs. 4(c) and 4(i)].

As shown in Figs. 4(c) and 4(i), the imaging of the targeted NPs alone is not ideal for differentiating between tumor (biomarker-positive) and normal (biomarker-negative) tissues due to the misleading nonspecific accumulation of the NPs. For example, the nonspecific accumulation of NPs is heavily influenced by mechanical properties of tissue such as porosity, which is often higher in normal tissues compared with dense tumors. ${ }^{15,17,28}$ In addition, the imaging of raw NP signals is sensitive to variations in illumination power and detector working distance (often due to tissue-surface irregularities). As an alternative, the ratiometric imaging of targeted versus untargeted NPs mitigates such misleading nonspecific effects, and allows for the unambiguous identification of tumors based on their elevated biomarker expression [Figs. 4(d) and 4(j)]. Note that the ratiometric imaging approach has been widely adopted for many molecular imaging applications using various types of optical contrast agents such as SERS NPs and fluorescent agents. ${ }^{16,20,24,29-32}$ Among those contrast agents, SERRS NPs and SERS NPs are particularly well suited for ratiometric imaging due to the identical geometry and surface properties (same coating materials) of all of the NP flavors, and the fact that they can all be excited at a single illumination wavelength and imaged at the same wavelength range, ensuring that all NP flavors are interrogated identically in terms of optical irradiance and penetration depth.

For the tissue-imaging studies, the SERRS NPs allowed for the differentiation of tumor and normal tissue at the shortest integration times [10 and $2 \mathrm{~ms}$; Figs. 4(e) and 4(f)], whereas 
the SERS NPs failed to do so [Figs. 4(k) and 4(1)]. Even at the shortest integration time of $2 \mathrm{~ms}$, the SERRS NP signals exhibited high signal-to-noise ratios [Fig. 4(n)] and small measurement errors $[< \pm 20 \%$, Fig. 4(o)]. Assuming that an error in the NP ratio of $< \pm 20 \%$ can allow for accurate identification of tumors, the use of SERRS NPs allows our system to image 60,000 pixels/min using a 6-mW laser power and a 1-ms integration time (the minimum integration time of our current CCD), which enables an imaging rate of $150-\mathrm{cm}^{2} / \mathrm{min}$ at a spatial resolution of $0.5 \mathrm{~mm}$. Note that this imaging speed is limited by the integration time of the CCD in our current spectrometer. However, there is sufficient signal strength from the SERRS NPs to theoretically increase the imaging speed by another order of magnitude $(\sim 10 \times)$ if another detector array, capable of a higher frame rate (shorter integration time), is used.

\section{Conclusions}

We have explored the use of SERRS NPs to enhance the imaging speed of a fiber-optic spectral imaging system for intraoperative examination of large surgical margins. The SERRS NPs were synthesized and conjugated to HER 2 antibodies to achieve a high binding affinity that is comparable with our previously developed HER2-targeted SERS NPs, as validated by flow cytometry [Figs. 3(a) and 3(b)]. With an identical tissue-staining and imaging protocol, the multiplexed HER2-targeted and isotype-control SERRS NPs enabled accurate identification of HER2-overexpressed tumor xenografts, with a 50-fold enhanced imaging speed compared with what is achievable using commercial SERS NPs (Fig. 4). This should enable our system to image tissue surfaces at a rate of $150 \mathrm{~cm}^{2} / \min$ at $500-\mu \mathrm{m}$ spatial resolution. In terms of potential clinical impact, the use of SERRS NPs should enable our system to comprehensively image the entire surgical margin of a large lumpectomy specimen that is $\sim 10 \mathrm{~cm}$ in diameter in $7 \mathrm{~min}$ ( $5 \mathrm{~min}$ for staining and 2 min for imaging). Note that a submillimeter spatial resolution was achieved in these studies, which has been shown to enable a tumor-detection sensitivity of $>90 \%$ in a recent clinical study. ${ }^{16}$ However, if intraoperative time constraints are relaxed, higher resolution imaging may be achieved [Fig. 3(d)], which could further improve tumor-detection sensitivity (especially for smaller lesions). In addition, the use of more SERRS NP flavors in the future can also improve the sensitivity to detect molecularly heterogeneous tumors.

\section{Disclosures}

Jonathan T. C. Liu has an issued patent, "Raman imaging devices and methods of molecular imaging." The other authors declare no competing financial interests.

\section{Acknowledgments}

The authors acknowledge support from the NIH/NIBIB R21 EB015016 (J. T. C. Liu), the NIH/NCI R21 CA215561 (J. T. C. Liu), the Department of Mechanical Engineering at the University of Washington, and the Department of Education GAANN fellowship program (S. Kang). The Northwest BioTrust (NWBT) is supported, in part, by the NIH (P30CA015704).

\section{References}

1. L. Jacobs, "Positive margins: the challenge continues for breast surgeons," Ann. Surg. Oncol. 15(5), 1271-1272 (2008).
2. O. Yossepowitch et al., "Positive surgical margins in radical prostatectomy: outlining the problem and its long-term consequences," Eur. Urol. 55(1), 87-99 (2009).

3. P. Ang, A. W. Tan, and C. L. Goh, "Comparison of completely versus incompletely excised cutaneous squamous cell carcinomas," Ann. Acad. Med. Singapore 33(1), 68-70 (2004).

4. S. Talbot and B. Hitchcock, "Incomplete primary excision of cutaneous basal and squamous cell carcinomas in the Bay of Plenty," N. Z. Med. J. 117(1192), U848 (2004).

5. R. Haque et al., "Surgical margins and survival after head and neck cancer surgery," BMC Ear Nose Throat Disord. 6, 2 (2006).

6. R. Weissleder et al., Molecular Imaging: Principles and Practice, People's Medical Publishing House, Shelton, Connecticut (2010).

7. A. Marusyk and K. Polyak, "Tumor heterogeneity: causes and consequences," Biochim. Biophys. Acta 1805(1), 105-117 (2010).

8. Y. Wang, B. Yan, and L. Chen, "SERS tags: novel optical nanoprobes for bioanalysis," Chem. Rev. 113(3), 1391-1428 (2013).

9. Y. W. Wang et al., "Multiplexed optical imaging of tumor-directed nanoparticles: a review of imaging systems and approaches," Nanotheranostics 1(4), 369-388 (2017).

10. C. L. Zavaleta et al., "A Raman-based endoscopic strategy for multiplexed molecular imaging," Proc. Natl. Acad. Sci. U.S.A. 110(25), E2288-E2297 (2013).

11. Y. W. Wang et al., "Multiplexed molecular imaging of fresh tissue surfaces enabled by convection-enhanced topical staining with SERScoded nanoparticles," Small 12(40), 5612-5621 (2016).

12. J. V. Jokerst et al., "Affibody-functionalized gold-silica nanoparticles for Raman molecular imaging of the epidermal growth factor receptor," Small 7(5), 625-633 (2011).

13. S. Pal et al., "MUC1 aptamer targeted SERS nanoprobes," Adv. Funct. Mater. 27(32), 1606632 (2017).

14. Y. Wang et al., "Quantitative molecular phenotyping with topically applied SERS nanoparticles for intraoperative guidance of breast cancer lumpectomy," Sci. Rep. 6, 21242 (2016).

15. Y. Wang et al., "Surgical guidance via multiplexed molecular imaging of fresh tissues labeled with SERS-coded nanoparticles," IEEE J. Sel. Top. Quantum Electron. 22(4), 154-164 (2016).

16. Y. W. Wang et al., "Raman-encoded molecular imaging with topically applied SERS nanoparticles for intraoperative guidance of lumpectomy," Cancer Res. 77(16), 4506-4516 (2017).

17. Y. W. Wang et al., "Rapid ratiometric biomarker detection with topically applied SERS nanoparticles," Technology 2(2), 118-132 (2014).

18. L. Sinha et al., "Quantification of the binding potential of cell-surface receptors in fresh excised specimens via dual-probe modeling of SERS nanoparticles," Sci. Rep. 5, 8582 (2015).

19. S. Harmsen et al., "Surface-enhanced resonance Raman scattering nanostars for high precision cancer imaging," Sci. Transl. Med. 7(271), 271 ra7 (2015).

20. A. Oseledchyk et al., "Folate-targeted surface-enhanced resonance Raman scattering nanoprobe ratiometry for detection of microscopic ovarian cancer," ACS Nano 11(2), 1488-1497 (2017).

21. S. Harmsen et al., "Rational design of a chalcogenopyrylium-based surface-enhanced resonance Raman scattering nanoprobe with attomolar sensitivity," Nat. Commun. 6, 6570 (2015).

22. S. Harmsen et al., "Cancer imaging using surface-enhanced resonance Raman scattering nanoparticles," Nat. Protocol 12(7), 1400-1414 (2017).

23. C. Graf et al., "Surface functionalization of silica nanoparticles supports colloidal stability in physiological media and facilitates internalization in cells," Langmuir 28(20), 7598-7613 (2012).

24. E. Garai et al., "High-sensitivity, real-time, ratiometric imaging of surface-enhanced Raman scattering nanoparticles with a clinically translatable Raman endoscope device," J. Biomed. Opt. 18(9), 096008 (2013).

25. D. Van de Sompel et al., "A hybrid least squares and principal component analysis algorithm for Raman spectroscopy," PLoS One 7(6), e38850 (2012).

26. Y. W. Wang et al., "In vivo multiplexed molecular imaging of esophageal cancer via spectral endoscopy of topically applied SERS nanoparticles," Biomed. Opt. Express 6(10), 3714-3723 (2015).

27. K. Subik et al., "The expression patterns of ER, PR, HER2, CK5/6, EGFR, Ki-67 and AR by immunohistochemical analysis in breast cancer cell lines," Breast Cancer: Basic Clin. Res. 4, 35-41 (2010). 
28. K. M. Tichauer et al., "Improved tumor contrast achieved by single time point dual-reporter fluorescence imaging," J. Biomed. Opt. 17(6), 066001 (2012).

29. R. J. Mallia et al., "Wide-field multiplexed imaging of EGFR-targeted cancers using topical application of NIR SERS nanoprobes," Nanomedicine 10(1), 89-101 (2015).

30. J. T. C. Liu et al., "Quantifying cell-surface biomarker expression in thick tissues with ratiometric three-dimensional microscopy," Biophys. J. 96(6), 2405-2414 (2009).

31. K. M. Tichauer et al., "Microscopic lymph node tumor burden quantified by macroscopic dual-tracer molecular imaging," Nat. Med. 20(11), 1348-1353 (2014).

32. C. W. Barth et al., "Optimizing fresh specimen staining for rapid identification of tumor biomarkers during surgery," Theranostics 7(19), 4722-4734 (2017).

Yu "Winston" Wang received his PhD in mechanical engineering from Beihang University, Beijing, China, in 2013. After graduation, he joined Dr. Jonathan Liu's Research Group as a postdoctoral associate to develop SERS nanoparticles and spectral imaging systems for visualizing cancer biomarkers in tissues. His research interests include biomedical optics and molecular imaging.

Qian Yang received her PhD in chemical biology from Sichuan University, Sichuan, China, in 2013. During 2015 to 2016, she worked in Dr. Jonathan Liu's Research Group as a visiting scholar to design and synthesize SERS nanoparticles for rapid visualization of cancer biomarkers in tissues. Currently, she is an associate professor in the School of Pharmacy at Chengdu Medical College (China), working in the field of targeted drug delivery and pharmaceutics.
Soyoung Kang received her BS degree in biomedical engineering from the University of Southern California in 2010 and her MS degree in medical systems engineering from the University of Magdeburg, Germany, in 2014. Currently, she is pursuing a PhD degree in the Mechanical Engineering Department at the University of Washington. She was a GAANN Fellow from 2014 to 2017. Her work aims to develop a Raman-imaging system for visualizing cancer biomarkers intraoperatively during tumor-resection procedures.

Matthew A. Wall received his PhD in chemistry from City University of New York in 2016. After graduation, he worked in Dr. Jonathan Liu's research group to design and synthesize SERRS and SERS nanoparticles for intraoperative molecular imaging of tumors. Currently, he is a postdoctoral researcher at the Institute for Systems Biology to develop transcriptional regulatory networks of cancer. He was a GAANN Fellow from 2011 to 2016. His current research interests include computational biology and bioinformatics.

Jonathan T. C. Liu received his $\mathrm{PhD}$ at Stanford in 2005 and was a postdoc/instructor in the Molecular Imaging Program at Stanford from 2005 to 2010. He directs the laboratory for molecular biophotonics, which develops optical strategies for improving the diagnosis and treatment of diseases. From 2010 to 2014 , he was an assistant professor at Stony Brook and is now an associate professor in mechanical engineering at the University of Washington, with an adjunct appointment in pathology. https://www.me.washington.edu/people/ faculty/jonathan_liu. 